\title{
Simultaneous Imaging of Cerebrovascular Structure and Function in Hypertensive Rats Using Synchrotron Radiation Angiography
}

\author{
Liping Wang ${ }^{1,2 t}$, Zhihao $\mathrm{Mu}^{2,3+}$, Xiaojie Lin ${ }^{2}$, Jieli Geng ${ }^{4}$, Ti Qiao Xiao ${ }^{5}$, Zhijun Zhang ${ }^{2}$, \\ Yongting Wang ${ }^{2}$, Yongjing Guan ${ }^{6 *}$ and Guo-Yuan Yang ${ }^{1,2 *}$ \\ ${ }^{1}$ Department of Neurology, Ruijin Hospital, School of Medicine, Shanghai Jiao Tong University, Shanghai, China, ${ }^{2}$ Med-X \\ Research Institute and School of Biomedical Engineering, Shanghai Jiao Tong University, Shanghai, China, ${ }^{3}$ Department of \\ Pathology and Pathophysiology, Faculty of Basic Medical Sciences, Kunming Medical University, Kunming, China, \\ ${ }^{4}$ Department of Neurology, Renji Hospital, School of Medicine, Shanghai Jiao Tong University, Shanghai, China, ${ }^{5}$ Shanghai \\ Synchrotron Radiation Facility, Shanghai Institute of Applied Physics, CAS, Shanghai, China, ${ }^{6}$ Department of Radiology, \\ Ruijin Hospital, School of Medicine, Shanghai Jiao Tong University, Shanghai, China
}

OPEN ACCESS

Edited by: Hanting Zhang,

West Virginia University, United States

Reviewed by:

Yuchuan Ding,

Wayne State University School of

Medicine, United States

Huanyu Dou,

Texas Tech University Health Sciences

Center, United States

Deep R. Sharma,

SUNY Downstate Medical Center,

United States

*Correspondence:

Yongjing Guan

yongjingg@yahoo.com

Guo-Yuan Yang

gyyang0626@163.com

${ }^{\dagger}$ These authors have contributed equally to this work.

Received: 13 March 2017 Accepted: 19 October 2017 Published: 02 November 2017

Citation:

Wang L, Mu Z, Lin X, Geng J, Xiao TQ, Zhang Z, Wang Y, Guan Y and Yang G-Y (2017) Simultaneous Imaging of Cerebrovascular Structure and Function in Hypertensive Rats

Using Synchrotron Radiation Angiography.

Front. Aging Neurosci. 9:359. doi: 10.3389/fnagi.2017.00359
Hypertension has a profound influence on the structure and function of blood vessels. Cerebral vessels undergo both structural and functional changes in hypertensive animals. However, dynamic changes of cerebrovasculature and the factors involved in this process are largely unknown. In this study, we explored the dynamic changes of vascular structure in hypertensive rats using novel synchrotron radiation angiography. Twenty-four spontaneously hypertensive rats (SHR) and 24 Sprague-Dawley (SD) rats underwent synchrotron radiation (SR) angiography. Each group had 8 animals. We studied the cerebral vascular changes in SHR over a time period of 3-12-month and performed quantitative analysis. No vascular morphology differences between SHR and SD rats were observed in the early stage of hypertension. The number of twisted blood vessels in the front brain significantly increased at the 9- and 12-month observation time-points in the SHR compared to the SD rats $(p<0.01)$. The vessel density of the cortex and the striatum in SHR was consistently higher than that in SD rats at time points of 3-, 9-, and 12-month $(\rho<0.001)$. Vascular elasticity decreased both in SHR and SD rats with aging. There were statistically significant differences in the relative vascular elasticity of extracranial/intracranial internal carotid artery, middle cerebral artery, posterior cerebral artery and anterior cerebral artery between SHR and SD rats at 12 -month $(p<0.01)$. We concluded that the dynamic vascular alterations detected by SR angiography provided novel imaging data for the study of hypertension in vivo. The longer the course of hypertension was, the more obvious the vascular differences between the SHR and the $\mathrm{SD}$ rats became.

Keywords: angiography, cerebral vessels, hypertension, synchrotron radiation, vascular elasticity

Abbreviations: ACA, anterior cerebral artery; CDU, color Doppler ultrasonography; CTA, CT angiography; DBP, diastolic blood pressure; DSA, digital subtraction angiography; ICA, internal carotid artery; MBP, mean blood pressure; MCA, middle cerebral artery; MRA, MR angiography; PCA, posterior cerebral artery; SBP, systolic blood pressure; SD rats, Sprague-Dawley rats; SHR, spontaneously hypertensive rats; SR, synchrotron radiation. 


\section{INTRODUCTION}

Hypertension is a complex disease resulting from many pathogenic factors and has an impact on nervous system diseases. Studies showed that a long history of hypertension was the key risk factor for stroke (Takahashi et al., 2011; Turin et al., 2016). Hypertension could cause cerebral small vessel disease, which induced white matter damage and cognitive decline (Kaiser et al., 2014). Hypertension also impaired neurogenesis and long-term memory (Shih et al., 2016). Previous studies showed that cerebral vessels underwent both structural and functional changes in hypertensive animals (Nordborg and Johansson, 1980; Winquist and Bohr, 1983; Hughes and Bund, 2002; Cates et al., 2012). Cerebrovascular remodeling was the most important pathological change during hypertension (Lin et al., 1990; Lee and Griendling, 2008). For example, cerebral arteries from spontaneously hypertensive rats (SHR) were less compliant than those of the control. The arterial media was thicker during established hypertension in SHR (Mulvany et al., 1978; Nordborg and Johansson, 1980). Impairment of bloodbrain barrier was induced by hypertension (Ueno et al., 2011; Biancardi and Stern, 2016). However, most of these studies were conducted using histology and immunohistochemistry in fixed brain or vascular tissue (Carnevale et al., 2012; Chan et al., 2015). Detecting dynamic changes of vascular morphology in living animals is a great challenge for neurologists and neurobiologists. Studies in vitro cannot detect the real pathological change of cerebral vessels. Current in vivo techniques for assessing vasculature and arterial functions still suffer from insufficient imaging resolution, making it difficult to detect subtle changes in biological specimens (Lin et al., 2013; Yuan et al., 2013). Little is known about the structural and functional changes of deep brain vessels in hypertensive animals (Morishita et al., 2006). Thus, developing a novel approach to dynamically and directly observe cerebrovascular alterations in vivo is necessary and timely, and could provide further insight into the pathology of vascular disorder in hypertensive animals.

Common devices used to visually study vascular morphology in living animals include optical imaging, CT angiography (CTA), MR angiography (MRA), digital subtraction angiography (DSA) and color Doppler ultrasonography (CDU) (Acar et al., 2015; Hoshikawa et al., 2016; Hsieh et al., 2016; Su et al., 2016; Xu et al., 2016; Chen et al., 2017). CTA and DSA are both $\mathrm{x}$-ray based imaging methods used to assess vascular abnormalities in clinical and animal studies (Walkoff et al., 2016; Sun et al., 2017). Highresolution MRA is used to measure vessel patency in the entire process longitudinally (Duong, 2012; Shih et al., 2012). However, the resolution of these methods is limited for the detection of microvasculature disorders in rodents, as they have difficulty in detecting changes of small vessels less than $500 \mu \mathrm{m}$ in diameter (Dehkharghani et al., 2015; Detorakis et al., 2015; Wu et al., 2015; Kampschulte et al., 2016). Synchrotron radiation (SR) xray has a wide continuous adjustability of energy, high brilliancy, narrow pulse, collimating coherence and monochromaticity ( $\mathrm{Hu}$ et al., 2014; Zhang et al., 2014). The spatial resolution of SR xray imaging can reach sub-micron, around 1,000 times higher than that of conventional X-Ray absorption imaging (Liu et al.,
2006; Zhang et al., 2014). Recent developed SR angiography showed that it could be used for high-resolution imaging of cerebral vasculature in rodents (Lin et al., 2013; Yuan et al., 2013; Xi et al., 2015; Mu et al., 2016). It provides a unique tool for monitoring real time hemodynamic changes in blood flow and microvascular morphology. Fuji et al. used SR angiography to explore the association between endothelial function and microvascular remodeling in pulmonary arterial hypertension (Fuji et al., 2016). Miya et al. made use of synchrotron radiation to visualize the renal arterioles, glomeruli, and proximal tubules of rats in vivo (Miya et al., 2017). By performing SR angiography, our previous studies demonstrated that netrin-1 overexpression in the mouse brain, and transplantation of endothelial progenitor cells promoted functional angiogenesis (Lu et al., 2012; Chen et al., 2014). In addition, SR angiography has also been used in the evaluation of cerebral vasospasm in subarachnoid hemorrhage and cerebrovascular changes in hyperglycemic rats (Cai et al., 2012; Mu et al., 2016).

In this study, we performed SR angiography in SHR and Sprague-Dawley (SD) rats to compare the differences both in vascular structure and function with aging. In consideration of parts where cerebral diseases such as stroke might occur, our study focused on the Willis circle, cortex and striatum (Lipton, 1999). We also explored whether alterations of the vascular structure were related to the blood pressure and age and thereby affected outcomes of vascular diseases.

\section{MATERIALS AND METHODS}

\section{Animals and Experimental Groups}

Animal procedures were reviewed and approved by the Institutional Animal Care and Use Committee (IACUC) of Shanghai Jiao Tong University, Shanghai, China. Adult male SHR $(n=24)$ and adult male SD rats $(n=24)$ weighing $250 \pm 30$ grams (Sippr-BK, Inc., Shanghai, China) were used in the study. The SHR were randomly divided into 3 groups of 8 referred to as 3-, 9-, and 12-month-old groups. The SD rats used as controls were randomly divided into 3 groups as well. Animals were fed normally in the SPF proved animal facility in Shanghai Jiao Tong University, China.

\section{Measurement of Blood Pressure}

Systolic blood pressure (SBP) and diastolic blood pressure (DBP) were measured every week using a non-invasive tail cuff acquisition method (BP-98A, Softron Beijing Incorporated, Beijing, China). Rats were placed in a restrainer to acclimate themselves. All rats were first acclimated to the blood pressure measurements for 3 days before the acquisition (Biancardi et al., 2014). Arterial blood pressures were derived from the average results of $>3$ measurements in each recording session. Both SBP and DBP were obtained and recorded for the data analysis.

\section{SR Angiography}

SR angiography was conducted at the X-ray imaging beam line BL13W in Shanghai Synchrotron Radiation Facility (SSRF). Imaging setup and procedures have been described previously (Guan et al., 2012; Yuan et al., 2014). Briefly, the average beam 
current was $145 \mathrm{~mA}$, and X-ray energy was $33.2 \mathrm{keV}$. Animals were anesthetized by using ketamine/xylazine $(100 \mathrm{mg} / 10 \mathrm{mg} / \mathrm{kg}$, intraperitoneally) during the imaging process. An angiographic tube formed by connecting a PE-10 tube with a PE-50 tube was inversely inserted into the external carotid artery to allow injection of a contrast agent. The rat was placed perpendicular to the beam on its left side, and $160 \mu$ l of diluted iodinated contrast medium, ipamiro (General Electric Company, Shanghai, China), was injected into the common carotid artery through the angiographic tube at a speed rate of $6 \mathrm{ml} / \mathrm{min}$ using an automated micro-syringe pump (LSP01-1A, Longer; Baoding, China). An $\mathrm{X}$-ray complementary metal oxide semiconductor (pixel size $13.0 \times 13.0 \mu \mathrm{m}$, frame frequency $30 \mathrm{~Hz}$, Hamamatsu Ltd., Japan) was used to record high-resolution real-time angiographic images. The X-ray complementary metal oxide semiconductor was positioned at a distance of $780 \mathrm{~mm}$ from the sample.

\section{Assessment of Vessel Morphology}

We used image analysis software (Image J, National Institutes of Health, Bethesda, MD) to conduct measurements of the internal diameter of vessels in the circus Willis. Vessel internal diameter presented was the average of three independent measurements. To assess cortical and striatal microvascular perfusion, we measured the vessel density using a perfusion angiogram. A specialized program written in Matlab (MathWorks, Natick, Massachusetts) was used to identify perfused vessels from original SR angiography images. The vessel signals in each area were summed.

To assess the number of curly small vessels, we selected the same area on each image and amplified the area to find bending parts of the small vessels, which presented high signal value, then calculated the number of the bending parts of each group.

\section{Calculation of Arterial Elasticity}

The theory and method of the elasticity calculation has been described previously (Lin et al., 2015). In the imaging procedure, sequential $x$-ray transmission images $I(x, y, t)$ of the rat brain were recorded. Relative vascular elasticity was estimated from the absorption maps $I_{a b s}(x, y, t)$.

$$
I_{a b s}(x, y, t)=\ln \left(I_{a b s}(x, y, 0)\right)-\ln \left(I_{a b s}(x, y, t)\right)
$$

The blood vessels were then manually segmented, and the diameter $r_{0}$ of each vessel was calculated for each ridge point. The absorption data of the cross-sectional line at each ridge point in $I_{a b s}(x, y, t)$ was extracted based on the perpendicular relationship between the ridge and cross-section. The spatiotemporal dynamics $I_{a b s}(x, y, t)$ of each cross-section were constructed from the image sequences $I_{a b s}(x, y, t)$. In this study, the spatio-temporal dynamics of the contrast agent in $I_{r t}(r, t)(t$ $<t_{0}$ ) were fitted to a Gaussian surface (Equation 2).

$$
I_{r t}(r, t)=a \cdot e^{-\left(\left(r-r_{0} / 2\right)^{2} / \sigma_{r}^{2}+\left(t-t_{0}\right)^{2} / \sigma_{t}^{2}\right)}+b
$$

After estimating the fitting parameters in Equation (2), the relative velocity $\mathrm{v}$ of each cross-section was calculated using
Equation (3)

$$
v=\int_{0}^{t_{0}} \int_{-\frac{r_{0}}{2}}^{\frac{r_{0}}{2}} \frac{I_{r t}(r, t)}{\mathrm{t}_{0} r_{0}} d r d t
$$

The elasticity $\mathrm{E}$ of each cross-section was calculated using Equation (4).

$$
E=\frac{v}{r_{0}}
$$

\section{Hematoxylin and Eosin Staining}

Rats were anesthetized after SRA and perfused with saline followed by $4 \%$ paraformaldehyde transcardially. The brains were fixed in $4 \%$ paraformaldehyde for $24 \mathrm{~h}$ and dehydrated with a graded alcohol system and then embedded in paraffin. A series of 8- $\mu \mathrm{m}$-thick coronal sections were prepared for hematoxylin and eosin staining according to a previously reported study (Lin et al., 2013).

\section{Statistical Analysis}

Data were presented as mean \pm standard deviation (SD). Blood pressure, the number of curly vessels, and vascular elasticity among different groups were compared with an unpaired Student t-test using SPSS 16.0 (SPSS Inc., Armonk, NY). One-way ANOVA was carried out to assess within-group differences of arterial elasticity, followed by a 2 -tailed Student $t$-test to determine statistical significance. A probability value of $p<0.05$ was considered significant.

\section{RESULTS}

\section{Sustained Hypertension in SHR Over Time}

We measured the blood pressure of both SHR and SD rats. Blood pressure in SHR and SD rats both remained stable from the 3rd-12th month. Meanwhile SBP, DBP, and mean blood pressure (MBP) were significantly elevated in the SHR 3-, 9-, and 12month groups compared to the age-matched SD rats $(p<0.001)$. The mortality of SHR was less $10 \%$ at the 12 -month observation point. This demonstrated that the blood pressure of the SHR we used in the study was at a relatively stable level (Figure 1), and thus this animal model was suitable for the vascular study.

\section{Increase of Curly Small Vessels in SHR}

To study the dynamic effect of hypertension on small intracranial vessels, we performed SRA to explore the structural alterations of the cerebral vessels. We found that the morphology of small vessels in the striatal area became disordered. Twisted small vessels manifested, and the curvature of the terminal branches significantly increased with time (Figure 2). We semi-quantified the number of intracranial curly small vessels in the brains of SHR and SD rats. The results showed that there was no significant difference in the number of curly small vessels between SHR and SD rats in the 3 -month groups (1.24 \pm 1.55 vs. $1.12 \pm$ $1.23, p>0.05)$. However, when hypertension was sustained for 9 months, the number of curly small vessels in the brain of SHR was significantly increased compared to those in the SD rats 


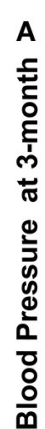

둘

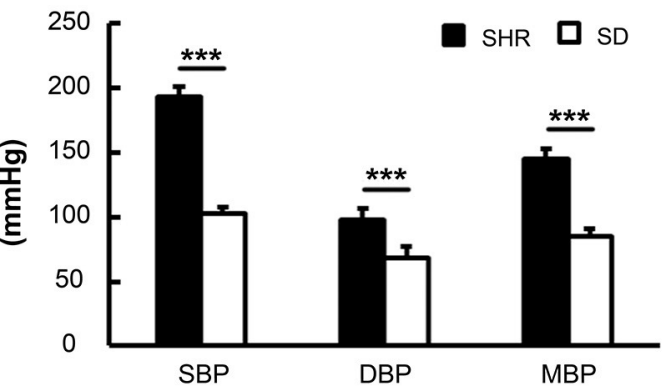

B

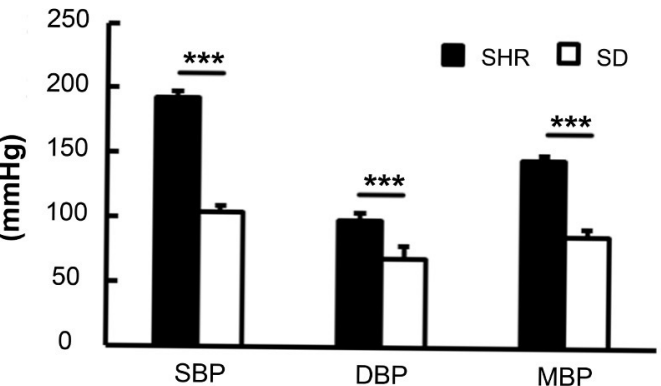

C

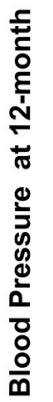

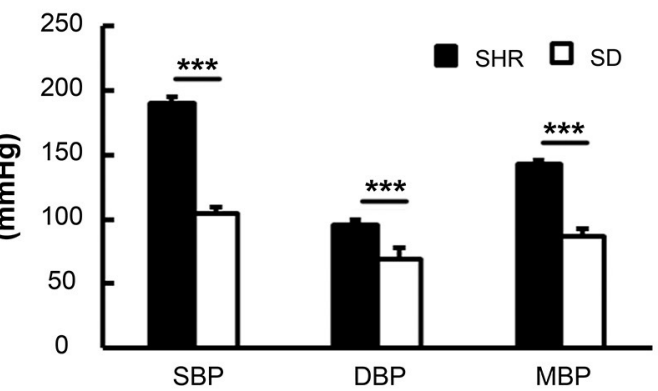

FIGURE 1 | Blood pressure of SHR was higher than SD rats in 3-(A), 9-(B), and 12-month (C). SBP, systolic blood pressure; DBP, diastolic blood pressure; MBP, mean blood pressure. ${ }^{\star \star \star} p<0.001$.

(Figure 3, $26.93 \pm 3.86$ vs. $15.38 \pm 3.46, p<0.001$ ). Similarly, an increase of vascular curvature was not evident in the early stage of hypertension. In later hypertension this change became obvious, and the quantity of the curly small vessels increased with time.

\section{Increased Vascular Density in SHR}

Using the Matlab software, we calculated the vascular density in the cortex and striatum from the original angiographic images. Perfused small vessel density in the cortex $(45.6 \pm 9.7,41.9$ \pm 6.7 , and $54.5 \pm 9.1 \%$ vs. $3.5 \pm 0.5 \%)$ and striatum $(44.4 \pm$ $7.3,47.1 \pm 7.0$, and $30.7 \pm 8.6$ vs. $5.6 \pm 0.8 \%$ ) of SHR was consistently higher in the 3-, 9-, and 12-month groups than that in the SD rats (Figure 4, $p<0.001$ ). Cerebral microcirculation perfusion volume was increased in SHR, but there was no difference in microcirculation perfusion in different time groups of SHR, indicating that hypertension could not further affect the microcirculation blood flow. Enhanced perfused small vessel

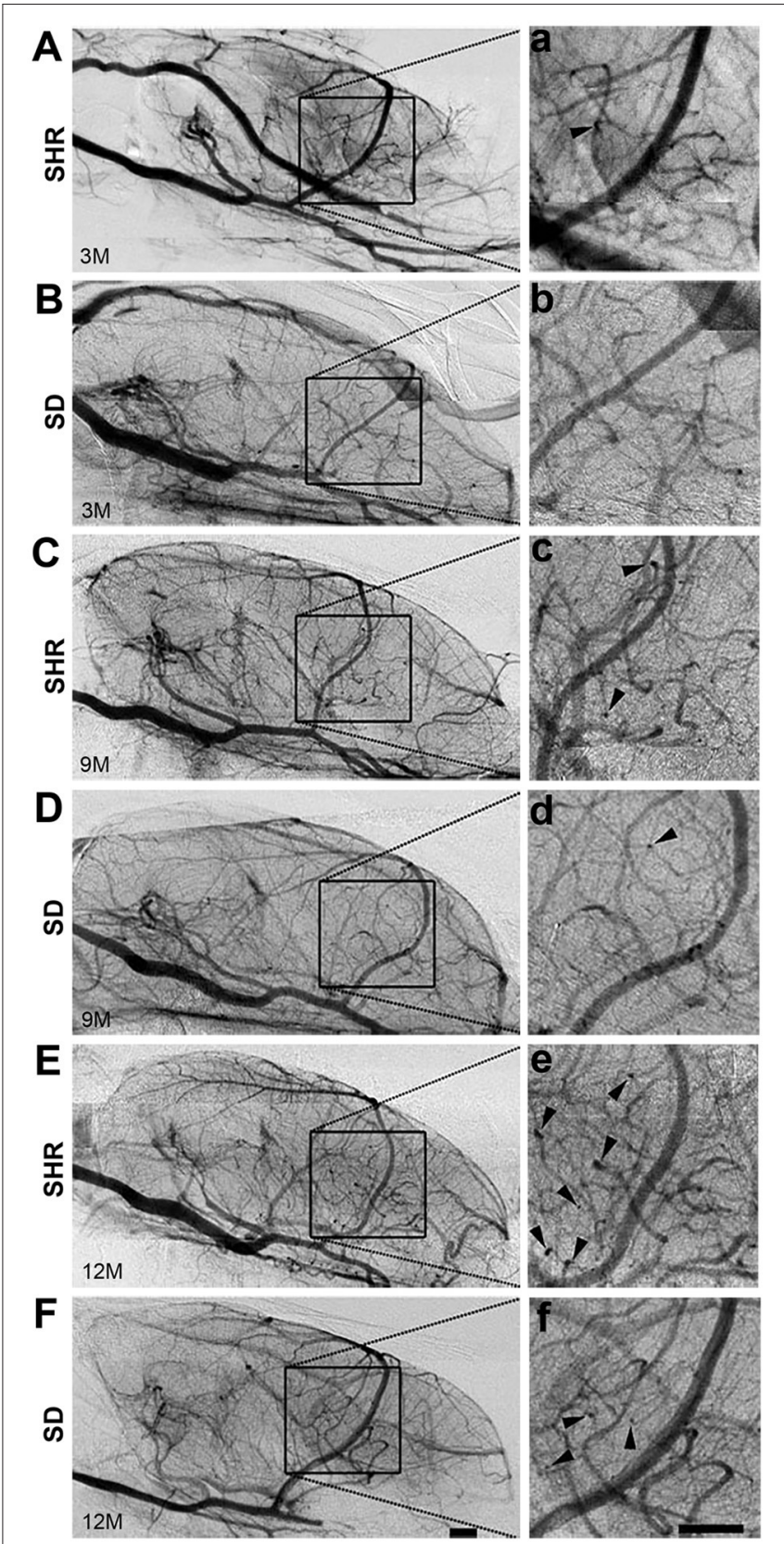

FIGURE 2 | Curly vessels in the brain of SHR increased significantly than SD rats. Angiography pictures were presented from SHR (A,C,E) and SD (B,D,F) rats in 3-, 9-, and 12-month. The images in square frames of graphs (a-f) were amplified as images (A-F), respectively. Square frame defines the selected area for calculating. Arrowhead points to curly vessels. Scale bar $=1 \mathrm{~mm}$.

density could be the result of an increase in the number of open small vessels, induced by hypertension.

\section{Reduction of Vascular Elasticity with Increased Duration of Hypertension}

To investigate the dynamic effects of hypertension on the intracranial large vessels, we calculated the vascular elasticity of all branches of Circus Willis using functional SR angiography. 


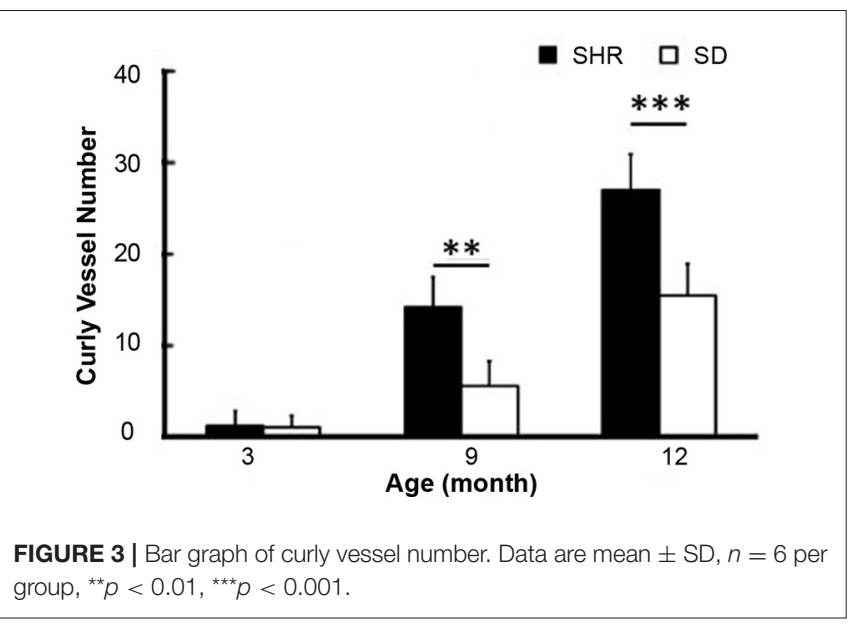

The elasticity values were normalized for comparison. The results showed that the vascular elasticity of SHR and SD rats decreased with aging, but there was a greater decrease in the SHR compared to the SD rats. The relative vascular elasticity of extracranial ICA of the SHR was lower than that of SD rats at the 9month time point $(0.28 \pm 0.02$ vs. $0.33 \pm 0.03, p<0.01)$. There were no significant differences in relative vascular elasticity of extracranial ICA, intracranial ICA, middle cerebral artery (MCA), posterior cerebral artery (PCA) and anterior cerebral artery (ACA) between the SHR and the SD rats at 9-month. It was noted that the differences of extracranial ICA, intracranial ICA, MCA, PCA and ACA between the SHR and the SD rats were significant at 12-month (Figure 5, $0.11 \pm 0.02$ vs. $0.18 \pm$ $0.02,0.12 \pm 0.03$ vs. $0.19 \pm 0.03,0.18 \pm 0.02$ vs. $0.23 \pm 0.03,0.17$ \pm 0.03 vs. $0.25 \pm 0.02,0.15 \pm 0.03$ vs. $0.24 \pm 0.03, p<0.001)$. Our results suggested that reduced vascular elasticity was a longterm effect of hypertension but did not occur after a short period of hypertension, as in the early stage of hypertension vascular elasticity was not affected. We only began to detect impairment in vascular elasticity caused by hypertension after the 9th month.

\section{Hypertension Induced Arterial Stenosis in ICA}

Interestingly, comparing the diameter of arteries between the SHR and the SD rats, we found that the diameter of the intracranial ICA but not the extracranial ICA, MCA or ACA, was narrowed in SHR in SR angiography images (Figure 6, 0.10 \pm 0.04 vs. $0.19 \pm 0.05, p<0.01$ ). The diameter of the ICA did not recover over the duration of study, suggesting that this phenomenon of intracranial ICA stenosis was permanent.

\section{DISCUSSION}

Using novel SR angiography technique, we first demonstrated dynamically in living SHR that: (1) the morphology of cerebral vessels gradually became twisted and multicurled with hypertension; (2) small vessel density was increased in the forebrain of SHR compared to that in the SD rats; (3) stenosis appeared in the intracranial ICA in SHR; and (4) the vascular

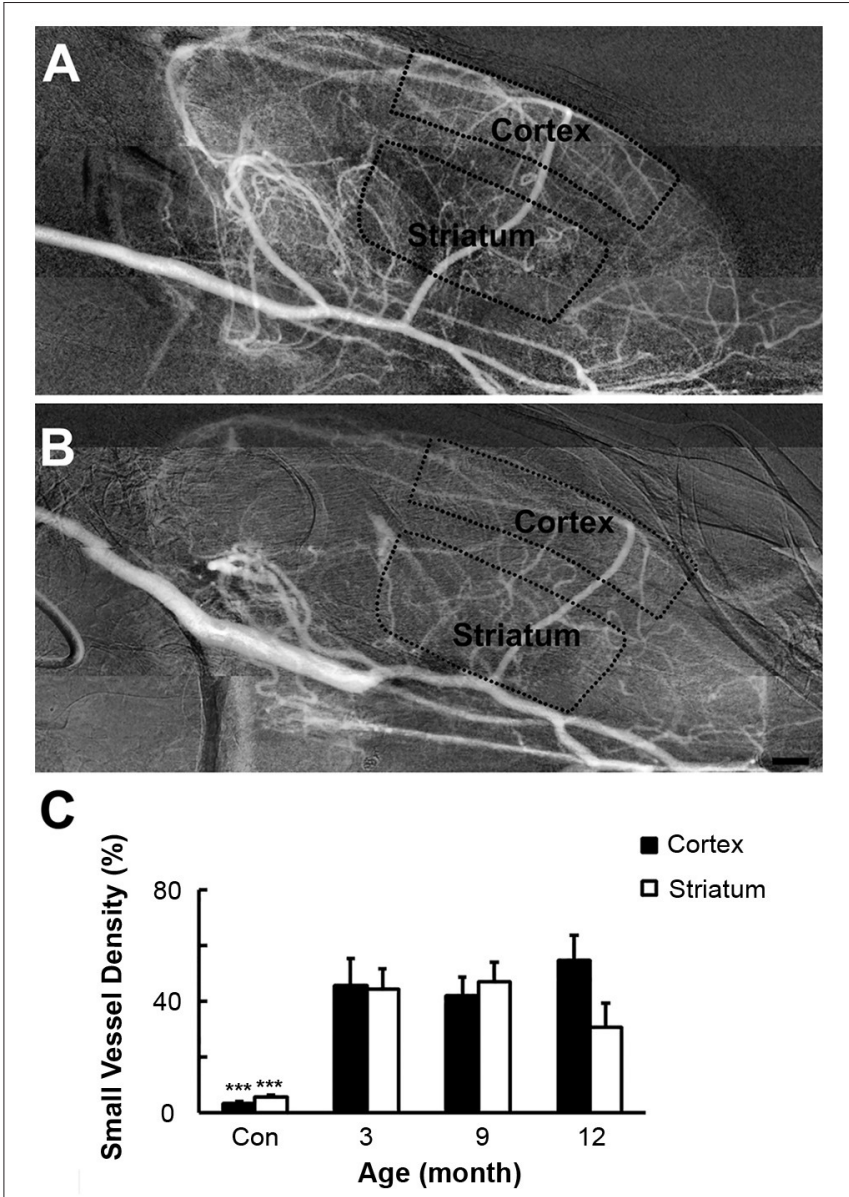

FIGURE 4 | Vascular density of SHR and SD rats. SHR (A) had increased perfused small vessel density in cortex and striatum than SD rats (B) at 3-, 9-, and 12-month. (C) Bar graph of small vessel density. Bar $=1 \mathrm{~mm}$. Data are mean $\pm \mathrm{SD}, n=6$ per group, ${ }^{\star \star \star} p<0.001$.

elasticity of the branches in Willis Circus decreased with hypertension.

Using SR angiography, a unique technique in the imaging field, we showed dynamic vascular structural alteration induced by hypertension in vivo. We found that the vascular structure became abnormal in the forebrain, especially in the MCA territory, with hypertension. There were obviously more tortuous vessels, and increased curvature of terminal branches in the SHR. Though there was no significant difference between SHR and SD rats at 3-month, the number of curly vessels at 9-, and 12-month was significantly increased in the SHR compared to the SD rats. The development of hypertension in the SHR had different effects on the vascular resistance of different parts of the vascular bed (Faraci and Heistad, 1990). The large arteries were affected at the early stage of hypertension. When hypertension was maintained at a certain level for a period of time, small and micro arteries carried more vascular resistance (Bohlen, 1989). Morphological abnormality in the SHR indicated that small cerebral vessels were affected by sustained hypertension. These multicurled small vessels could affect the function of 


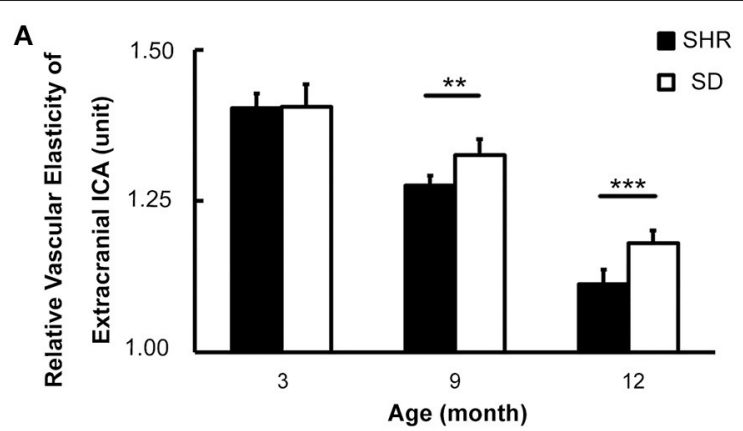

C

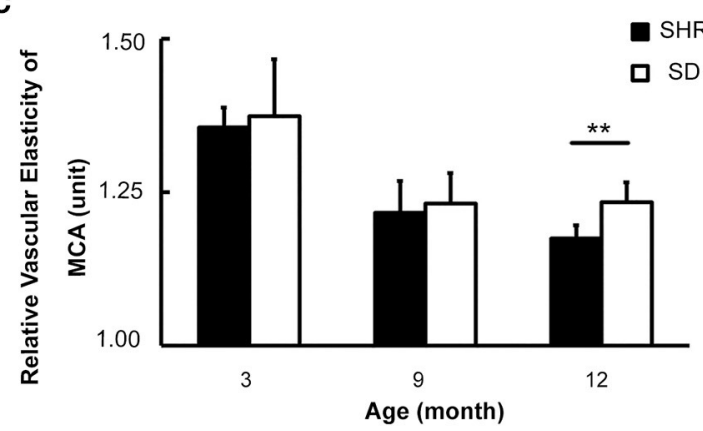

E

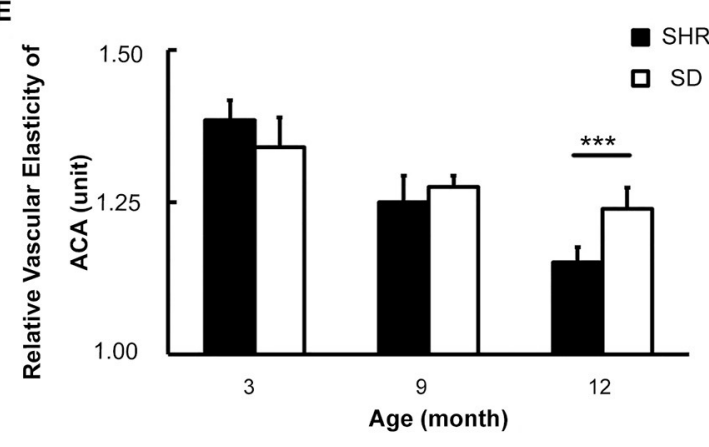

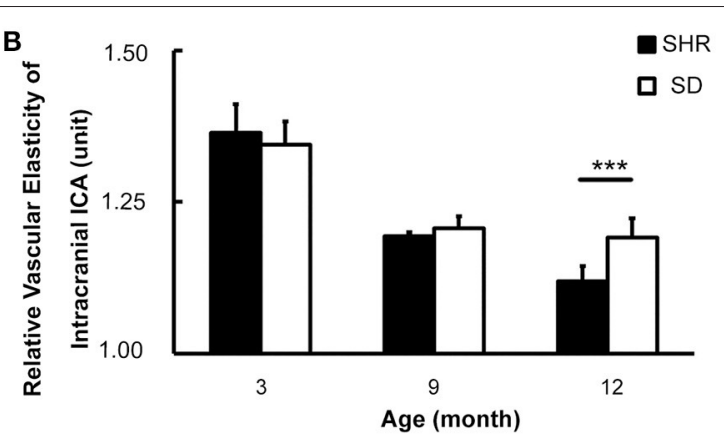

D

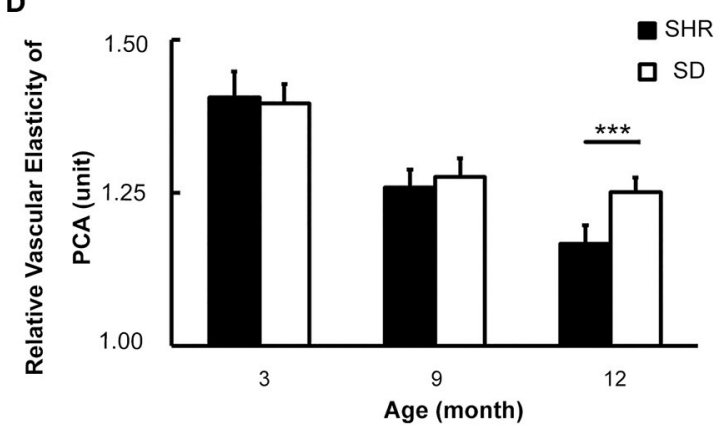

F

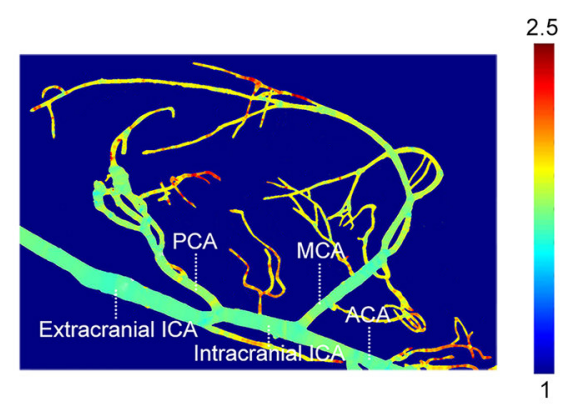

FIGURE 5 | Circus Willis of SHR had decreased vascular elasticity with the duration of hypertension increased. (A-E) Bar graph of relative vascular elasticity of extracranial ICA, intracranial ICA, MCA, PCA, and ACA in 3-, 9-, 12-month respectively. (F) Pseudocolor image demonstrated the vascular elasticity in extracranial ICA, intracranial ICA, MCA, PCA, and ACA. Data are mean $\pm \mathrm{SD}, n=6$ per group, ${ }^{\star *} p<0.01,{ }^{* * *} p<0.001$.

cerebral blood circulation and thus further impact on the development and prognosis of vascular disorder, contributing to the occurrence of stroke.

SR angiography results also showed that small vessel density was increased in the cortex and striatum of the SHR compared to those of the SD rats at 3-, 9-, and 12- month. Vessel density is an indicator of microvascular perfusion. Immunohistochemical staining of cerebral blood vessels could only reflect the number of blood vessels, not the exact number of the perfused microvessels. SR angiography is the only way to present cerebral microcirculation perfusion.

Data analysis suggested that hypertension could increase the functional capacity of capillaries. However, whether this increased capillary capacity could alleviate the insufficient blood supply during ischemic stroke needs to be further explored. Finally, we found intracranial ICA stenosis in SHR, suggesting that the intracranial ICA underwent reconstruction to adapt to hypertension. Remodeling induced by hypertension contributes to decreased diameter and increased wall-lumen ratio of vessels (Mulvany et al., 1996; Bonacasa et al., 2008; Kumai et al., 2008; Pires et al., 2010), which could reduce cerebral blood flow after ischemic stroke and affect recovery.

The functional alteration of cerebral vessels was induced by hypertension. Vascular elasticity is an indicator of vascular function (Fabiani et al., 2014; Turk et al., 2015). The functional status of the vasculature could therefore be predicted by vascular elasticity. We found that vascular elasticity of the branches in Circus Willis decreased with sustained hypertension. We noted that in the early stage of hypertension, there was no difference in vascular elasticity between the SHR and the SD rats. However, decreased vascular elasticity was associated with hypertension. Moreover, reduced vessel elasticity could cause vascular wall stiffness, which is the most important factor for hemorrhagic stroke. 

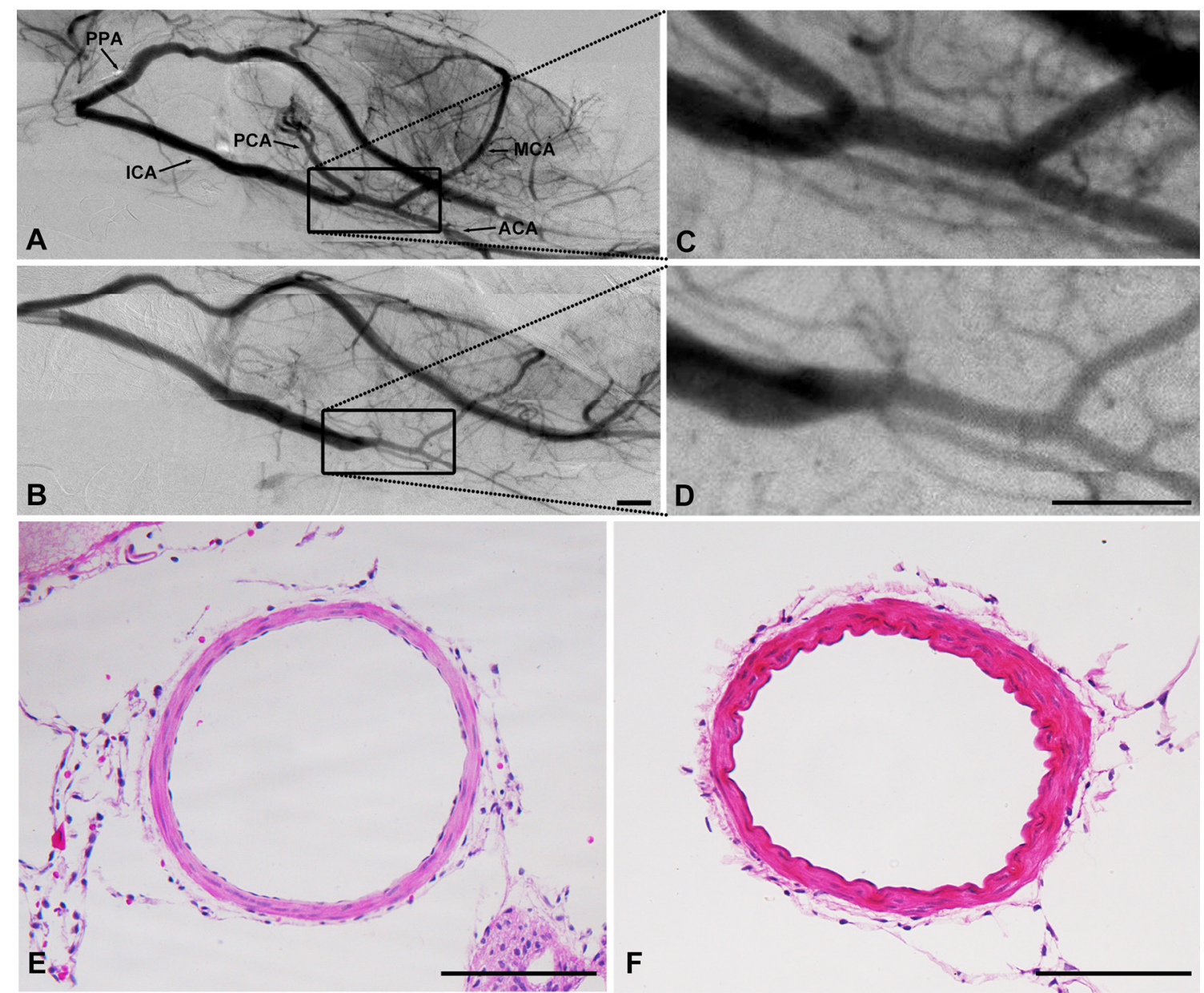

$F$

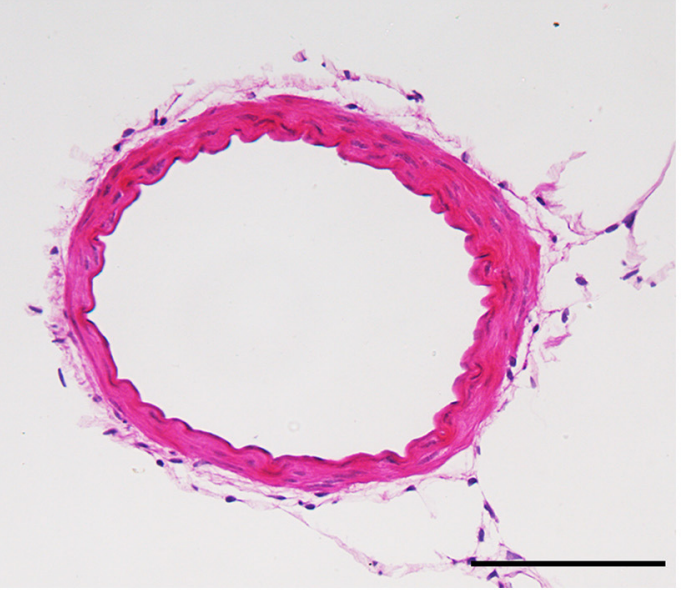

FIGURE 6 | SHR had arterial stenosis in ICA. Cerebral vascular morphology imaged by SR angiography in SHR (B) and SD rats (A). (C,D) were higher amplification. HE result showed that the diameter decreased while the wall-lumen ration of ICA increased in SHR (F) than that in SD rats (E). (A-D): Scale bar = $1 \mathrm{~mm}$. (E,F): Scale bar $=100 \mu \mathrm{m}$.

Functional SR angiography technique provides a novel noninvasive technique for detecting vascular elasticity. Compared to other methods of measuring cerebral vascular elasticity, functional SR angiography produces less damage (Regrigny et al., 2000). The method we developed could be used to monitor dynamic vascular function. Structural and functional alterations could be viewed in a single contrast image. Dynamic measurement of vascular elasticity in vivo is characteristic of functional SR angiography. This method is applicable to the measurement of deep blood vessels, which could not be detected by ultrasound elastography (Li et al., 2016). Although MRI is suitable for in vivo imaging, the lower resolution limits its use in the detection of small vessel changes. The slow imaging speed of MR is not suitable for dynamic imaging in living animals (Hughes et al., 2015). At the same time, we also showed that SR angiography could achieve real-time dynamic monitoring of vascular morphology in the rodent brain. We did not detect any difference in vasculature between the SHR and the SD rats using MRI and MRA (data not shown), which supported the fact that MRA was not a sensitive enough approach when exploring the structural and functional changes in cerebral vessels due to hypertension because of the limited resolution (Yang et al., 2008; Guan et al., 2012).

There were some limitations in our study. A previous study showed that aging is also associated with changes in vascular structural and cellular function in the brain (Chowdhury et al., 2011). As most clinical hypertension patients are older than 65 years old (Marini et al., 2001), researches on aged rats or mice are of great importance. In our study, we only explored vascular changes of SHR at 3-, 9-, and 12-month. Three-month represented the early stage of hypertension. The 6-month time point was skipped because the data of this time point resembled that of the 9-month time point in the preliminary study. The vascular changes of SHR at 24-month which could be referred to as aged rats still need to be explored (Tang et al., 2014). Compared to other studies using 1-5 months old animals, our study lasted longer (Coyle and Jokelainen, 1983; Winquist and Bohr, 1983; Kitamura et al., 2016; Stanzione et al., 2017; Takesue et al., 2017). Besides, the relationship between the vascular alterations including multicurled vessels, vessel density, 
vascular elasticity, and prognosis of stroke remains unclear. The molecular mechanism of vascular changes needs to be further investigated.

Deeper mechanisms of structural and functional alterations of cerebral vessels in hypertension remain to be studied further. Our in vivo dynamic study of living SHR at different stages of age could contribute to hypertension research. Hypertension can often cause pathological changes in the cerebrovasculature, and therefore monitoring intracranial vessel morphology and vascular function appears to be extremely important. SR angiography has great potential for experimental application, as this technique of fast simultaneous imaging of vascular morphology and elasticity can help to evaluate vascular function status.

In conclusion, our data directly demonstrated the structural and functional alterations of cerebral vessels induced by hypertension in vivo, indicating that functional SR angiography is a unique tool for detecting vascular disorder in rodents, which may benefit both clinical diagnosis and therapy.

\section{REFERENCES}

Acar, O., Esen, T., Colakoglu, B., Camli, M. F., and Cakmak, Y. O. (2015). Improving testicular blood flow with electroacupuncture-like percutaneous nerve stimulation in an experimental rat model of testicular torsion. Neuromodulation 18, 324-328; discussion328. doi: 10.1111/ner. 12246

Biancardi, V. C., and Stern, J. E. (2016). Compromised blood-brain barrier permeability: novel mechanism by which circulating angiotensin II signals to sympathoexcitatory centres during hypertension. J. Physiol. 594, 1591-1600. doi: 10.1113/JP271584

Biancardi, V. C., Son, S. J., Ahmadi, S., Filosa, J. A., and Stern, J. E. (2014). Circulating angiotensin II gains access to the hypothalamus and brain stem during hypertension via breakdown of the blood-brain barrier. Hypertension 63, 572-579. doi: 10.1161/HYPERTENSIONAHA.113.01743

Bohlen, H. G. (1989). The microcirculation in hypertension. J. Hypertens 7(Suppl), S117-S124.

Bonacasa, B., Sanchez, M. L., Rodriguez, F., Lopez, B., Quesada, T., Fenoy, F. J., et al. (2008). 2-Methoxyestradiol attenuates hypertension and coronary vascular remodeling in spontaneously hypertensive rats. Maturitas 61, 310-316. doi: 10.1016/j.maturitas.2008.09.028

Cai, J., Sun, Y., Yuan, F., Chen, L., He, C., Bao, Y., et al. (2012). A novel intravital method to evaluate cerebral vasospasm in rat models of subarachnoid hemorrhage: a study with synchrotron radiation angiography. PLoS ONE 7:e33366. doi: 10.1371/journal.pone.0033366

Carnevale, D., Mascio, G., D’andrea, I., Fardella, V., Bell, R. D., Branchi, I., et al. (2012). Hypertension induces brain beta-amyloid accumulation, cognitive impairment, and memory deterioration through activation of receptor for advanced glycation end products in brain vasculature. Hypertension 60, 188-197. doi: 10.1161/HYPERTENSIONAHA.112.195511

Cates, M. J., Dickinson, C. J., Hart, E. C., and Paton, J. F. (2012). Neurogenic hypertension and elevated vertebrobasilar arterial resistance: is there a causative link? Curr. Hypertens. Rep. 14, 261-269. doi: 10.1007/s11906-012-0267-6.

Chan, S. L., Umesalma, S., and Baumbach, G. L. (2015). Epidermal growth factor receptor is critical for angiotensin II-mediated hypertrophy in cerebral arterioles. Hypertension 65, 806-812. doi: 10.1161/HYPERTENSIONAHA.114.04794

Chen, C., Lin, X., Wang, J., Tang, G., Mu, Z., Chen, X., et al. (2014). Effect of HMGB1 on the paracrine action of EPC promotes post-ischemic neovascularization in mice. Stem Cells 32, 2679-2689. doi: 10.1002/stem.1754

Chen, H., Liu, N., Li, Y., Wu, B., Su, Z., Zhu, G., et al. (2017). The role of dynamic contrast-enhanced MR in evaluating level III collateral circulation

\section{AUTHOR CONTRIBUTIONS}

LW and ZM contributed to the design of experiment and the draft of the manuscript. XL, JG, and ZZ contributed to the acquisition of the data. TX, YW, YG, and GY helped to design the study and revise the manuscript. All authors approved the final version of the manuscript.

\section{FUNDING}

This work is supported by National Natural Science Foundation of China \#81201214 (YG) and National Natural Science Foundation of China \#U1232205 (GY).

\section{ACKNOWLEDGMENTS}

We thank Yuqi Ren and Rongchang Chen from SSRF, Shanghai Institute of Applied Physics, CAS for their technical support and useful discussion.

in a rat model of acute ischemic stroke. Mol Neurobiol. 54, 2731-2738. doi: 10.1007/s12035-016-9844-Z

Chowdhury, M. H., Nagai, A., Bokura, H., Nakamura, E., Kobayashi, S., and Yamaguchi, S. (2011). Age-related changes in white matter lesions, hippocampal atrophy, and cerebral microbleeds in healthy subjects without major cerebrovascular risk factors. J. Stroke Cerebrovasc. Dis. 20, 302-309. doi: 10.1016/j.jstrokecerebrovasdis.2009.12.010

Coyle, P., and Jokelainen, P. T. (1983). Differential outcome to middle cerebral artery occlusion in spontaneously hypertensive stroke-prone rats (SHRSP) and Wistar Kyoto (WKY) rats. Stroke 14, 605-611. doi: 10.1161/01.STR.14.4.605

Dehkharghani, S., Qiu, D., Albin, L. S., and Saindane, A. M. (2015). Dose reduction in contrast-enhanced cervical mr angiography: field strength dependency of vascular signal intensity, contrast administration, and arteriographic quality. AJR Am. J. Roentgenol. 204, W701-W706. doi: 10.2214/AJR.14.13435

Detorakis, E. E., Foukarakis, E., Karavolias, G., and Dermitzakis, A. (2015). Cardiovascular magnetic resonance and computed tomography in the evaluation of aneurysmal coronary-cameral fistula. J. Radiol. Case Rep. 9, 10-21. doi: 10.3941/jrcr.v9i7.2305

Duong, T. Q. (2012). Multimodal MRI of experimental stroke. Transl. Stroke Res. 3, 8-15. doi: 10.1007/s12975-011-0140-y

Fabiani, M., Low, K. A., Tan, C. H., Zimmerman, B., Fletcher, M. A., SchneiderGarces, N., et al. (2014). Taking the pulse of aging: mapping pulse pressure and elasticity in cerebral arteries with optical methods. Psychophysiology 51, 1072-1088. doi: 10.1111/psyp. 12288

Faraci, F. M., and Heistad, D. D. (1990). Regulation of large cerebral arteries and cerebral microvascular pressure. Circ. Res. 66, 8-17. doi: 10.1161/01.RES.66.1.8

Fuji, S., Matsushita, S., Hyodo, K., Osaka, M., Sakamoto, H., Tanioka, K., et al. (2016). Association between endothelial function and micro-vascular remodeling measured by synchrotron radiation pulmonary micro-angiography in pulmonary arterial hypertension. Gen. Thorac. Cardiovasc. Surg. 64, 597-603. doi: 10.1007/s11748-016-0684-6

Guan, Y., Wang, Y., Yuan, F., Lu, H., Ren, Y., Xiao, T., et al. (2012). Effect of suture properties on stability of middle cerebral artery occlusion evaluated by synchrotron radiation angiography. Stroke 43, 888-891. doi: 10.1161/STROKEAHA.111.636456

Hoshikawa, R., Kawaguchi, H., Takuwa, H., Ikoma, Y., Tomita, Y., Unekawa, M., et al. (2016). Dynamic flow velocity mapping from fluorescent dye transit times in the brain surface microcirculation of anesthetized rats and mice. Microcirculation. 23, 416-425. doi: 10.1111/micc.12285

Hsieh, M. C., Tsai, C. Y., Liao, M. C., Yang, J. L., Su, C. H., and Chen, J. H. (2016). Quantitative susceptibility mapping-based microscopy of magnetic resonance venography (QSM-mMRV) for in vivo morphologically and functionally 
assessing cerebromicrovasculature in rat stroke model. PLoS ONE 11:e0149602. doi: 10.1371/journal.pone.0149602

Hu, J., Cao, Y., Wu, T., Li, D., and Lu, H. (2014). High-resolution threedimensional visualization of the rat spinal cord microvasculature by synchrotron radiation micro-CT. Med. Phys. 41:101904. doi: $10.1118 / 1.4894704$

Hughes, J. D., Fattahi, N., Van Gompel, J., Arani, A., Meyer, F., Lanzino, G., et al. (2015). Higher-resolution magnetic resonance elastography in meningiomas to determine intratumoral consistency. Neurosurgery 77, 653-658; discussion 658-659. doi: 10.1227/NEU.0000000000000892

Hughes, J. M., and Bund, S. J. (2002). Arterial myogenic properties of the spontaneously hypertensive rat. Exp. Physiol. 87, 527-534. doi: 10.1113/eph8702399

Kaiser, D., Weise, G., Moller, K., Scheibe, J., Posel, C., Baasch, S., et al. (2014). Spontaneous white matter damage, cognitive decline and neuroinflammation in middle-aged hypertensive rats: an animal model of early-stage cerebral small vessel disease. Acta Neuropathol. Commun. 2:169. doi: 10.1186/s40478-014-0169-8

Kampschulte, M., Krombach, G. A., Richards, D. C., Sender, J., Lips, K. S., Thormann, U., et al. (2016). Neovascularization of osteoporotic metaphyseal bone defects: A morphometric micro-CT study. Microvasc. Res. 105, 7-14. doi: 10.1016/j.mvr.2015.10.005

Kitamura, A., Saito, S., Maki, T., Oishi, N., Ayaki, T., Hattori, Y., et al. (2016). Gradual cerebral hypoperfusion in spontaneously hypertensive rats induces slowly evolving white matter abnormalities and impairs working memory. J. Cereb. Blood Flow Metab. 36, 1592-1602. doi: 10.1177/0271678X15 606717

Kumai, Y., Ooboshi, H., Ago, T., Ishikawa, E., Takada, J., Kamouchi, M., et al. (2008). Protective effects of angiotensin II type 1 receptor blocker on cerebral circulation independent of blood pressure. Exp. Neurol. 210, 441-448. doi: 10.1016/j.expneurol.2007.11.028

Lee, M. Y., and Griendling, K. K. (2008). Redox signaling, vascular function, and hypertension. Antioxid. Redox Signal. 10, 1045-1059. doi: $10.1089 /$ ars.2007.1986

Li, Z., Du, L., Wang, F., and Luo, X. (2016). Assessment of the arterial stiffness in patients with acute ischemic stroke using longitudinal elasticity modulus measurements obtained with Shear Wave Elastography. Med. Ultrason. 18, 182-189. doi: 10.11152/mu.2013.2066.182.wav

Lin, S. Z., Sposito, N., Pettersen, S., Rybacki, L., Mckenna, E., Pettigrew, K., et al. (1990). Cerebral capillary bed structure of normotensive and chronically hypertensive rats. Microvasc. Res. 40, 341-357. doi: 10.1016/0026-2862(90)90032-M

Lin, X., Miao, P., Mu, Z., Jiang, Z., Lu, Y., Guan, Y., et al. (2015). Development of functional in vivo imaging of cerebral lenticulostriate artery using novel synchrotron radiation angiography. Phys. Med. Biol. 60, 1655-1665. doi: 10.1088/0031-9155/60/4/1655

Lin, X., Miao, P., Wang, J., Yuan, F., Guan, Y., Tang, Y., et al. (2013). Surgery-related thrombosis critically affects the brain infarct volume in mice following transient middle cerebral artery occlusion. PLoS ONE 8:e75561. doi: 10.1371/journal.pone.0075561

Lipton, P. (1999). Ischemic Cell Death in Brain Neurons. Physiol. Rev. 79, 1431-1568.

Liu, P., Sun, J., Guan, Y., Zhang, G., and Xu, L. X. (2006). Detection of lung cancer with phase-contrast X-ray imaging using synchrotron radiation. Conf. Proc. IEEE Eng. Med. Biol. Soc. 1, 2001-2004. doi: 10.1109/IEMBS.2006. 260802

Lu, H., Wang, Y., He, X., Yuan, F., Lin, X., Xie, B., et al. (2012). Netrin1 hyperexpression in mouse brain promotes angiogenesis and long-term neurological recovery after transient focal ischemia. Stroke 43, 838-843. doi: 10.1161/STROKEAHA.111.635235

Marini, C., Triggiani, L., Cimini, N., Ciancarelli, I., De Santis, F., Russo, T., et al. (2001). Proportion of older people in the community as a predictor of increasing stroke incidence. Neuroepidemiology 20, 91-95. doi: 10.1159/000054766

Miya, K., Matsushita, S., Hyodo, K., Tokunaga, C., Sakamoto, H., Mizutani, T., et al. (2017). Renal contrast microangiography with synchrotron radiation: a novel method for visualizing structures within nephrons in vivo. Acta Radiol. 58, 505-510. doi: 10.1177/0284185116658685
Morishita, A., Kondoh, T., Sakurai, T., Ikeda, M., Bhattacharjee, A. K., Nakajima, S., et al. (2006). Quantification of distension in rat cerebral perforating arteries. Neuroreport 17, 1549-1553. doi: 10.1097/01.wnr.0000234756.96183.29

Mu, Z. H., Jiang, Z., Lin, X. J., Wang, L. P., Xi, Y., Zhang, Z. J., et al. (2016). Vessel dilation attenuates endothelial dysfunction following middle cerebral artery occlusion in hyperglycemic rats. CNS Neurosci. Ther. 22, 316-324. doi: $10.1111 / \mathrm{cns} .12500$

Mulvany, M. J., Baumbach, G. L., Aalkjaer, C., Heagerty, A. M., Korsgaard, N., Schiffrin, E. L., et al. (1996). Vascular remodeling. Hypertension 28, 505-506.

Mulvany, M. J., Hansen, O. K., and Aalkjaer, C. (1978). Direct evidence that the greater contractility of resistance vessels in spontaneously hypertensive rats is associated with a narrowed lumen, a thickened media, and an increased number of smooth muscle cell layers. Circ. Res. 43, 854-864. doi: 10.1161/01.RES.43.6.854

Nordborg, C., and Johansson, B. B. (1980). Morphometric study on cerebral vessels in spontaneously hypertensive rats. Stroke 11, 266-270. doi: 10.1161/01.STR.11.3.266

Pires, P. W., Deutsch, C., Mcclain, J. L., Rogers, C. T., and Dorrance, A. M. (2010). Tempol, a superoxide dismutase mimetic, prevents cerebral vessel remodeling in hypertensive rats. Microvasc. Res. 80, 445-452. doi: 10.1016/j.mvr.2010.06.004

Regrigny, O., Atkinson, J., Capdeville-Atkinson, C., Liminana, P., and Chillon, J. M. (2000). Effect of lovastatin on cerebral circulation in spontaneously hypertensive rats. Hypertension 35, 1105-1110. doi: 10.1161/01.HYP.35.5.1105

Shih, Y. H., Tsai, S. F., Huang, S. H., Chiang, Y. T., Hughes, M. W., Wu, S. Y., et al. (2016). Hypertension impairs hippocampus-related adult neurogenesis, CA1 neuron dendritic arborization and long-term memory. Neuroscience 322, 346-357. doi: 10.1016/j.neuroscience.2016.02.045

Shih, Y. Y., Muir, E. R., Li, G., De La Garza, B. H., and Duong, T. Q. (2012). Highresolution 3D MR microangiography of the rat ocular circulation. Radiology 264, 234-241. doi: 10.1148/radiol.12112033

Stanzione, R., Bianchi, F., Cotugno, M., Marchitti, S., Forte, M., Busceti, C., et al. (2017). A decrease of brain microRNA-122 level is an early marker of cerebrovascular disease in the stroke-prone spontaneously hypertensive rat. Oxid. Med. Cell. Longev. 2017:1206420. doi: 10.1155/2017/1206420

Su, T., Wang, Y., Wang, J., Han, D., Ma, S., Cao, J., et al. (2016). In vivo magnetic resonance and fluorescence dual-modality imaging of tumor angiogenesis in rats using GEBP11 peptide targeted magnetic nanoparticles. J. Biomed. Nanotechnol. 12, 1011-1022. doi: 10.1166/jbn.2016.2233

Sun, L. J., Li, Y. D., Li, M. H., Wang, W., and Gu, B. X. (2017). Aneurysm outflow angle at MRA as discriminant for accurate diagnosis and differentiation between small sidewall cerebral aneurysms and infundibula. J. Neurointerv. Surg. 9, 591-594. doi: 10.1136/neurintsurg-2016-012425

Takahashi, I., Geyer, S. M., Nishi, N., Ohshita, T., Takahashi, T., Akahoshi, M., et al. (2011). Lifetime risk of stroke and impact of hypertension: estimates from the adult health study in Hiroshima and Nagasaki. Hypertens. Res. 34, 649-654. doi: 10.1038/hr.2011.7

Takesue, K., Kishi, T., Hirooka, Y., and Sunagawa, K. (2017). Activation of microglia within paraventricular nucleus of hypothalamus is NOT involved in maintenance of established hypertension. J. Cardiol. 69, 84-88. doi: 10.1016/j.jjcc.2016.01.004

Tang, Y., Wang, J., Lin, X., Wang, L., Shao, B., Jin, K., et al. (2014). Neural stem cell protects aged rat brain from ischemia-reperfusion injury through neurogenesis and angiogenesis. J. Cereb. Blood Flow Metab. 34, 1138-1147. doi: $10.1038 /$ jcbfm.2014.61

Turin, T. C., Okamura, T., Afzal, A. R., Rumana, N., Watanabe, M., Higashiyama, A., et al. (2016). Hypertension and lifetime risk of stroke. J. Hypertens. 34, 116-122. doi: 10.1097/HJH.0000000000000753

Turk, M., Pretnar-Oblak, J., Zupan, M., Zvan, B., and Zaletel, M. (2015). Ultrasound diagnosis of carotid artery stiffness in patients with ischemic leukoaraiosis. Ultrasound Med. Biol. 41, 64-71. doi: 10.1016/j.ultrasmedbio.2014.08.002

Ueno, M., Nakagawa, T., Nagai, Y., Nishi, N., Kusaka, T., Kanenishi, K., et al. (2011). The expression of CD36 in vessels with blood-brain barrier impairment in a stroke-prone hypertensive model. Neuropathol. Appl. Neurobiol. 37, 727-737. doi: 10.1111/j.1365-2990.2011.01172.x

Walkoff, L., Brinjikji, W., Rouchaud, A., Caroff, J., and Kallmes, D. F. (2016). Comparing magnetic resonance angiography (MRA) and computed 
tomography angiography (CTA) with conventional angiography in the detection of distal territory cerebral mycotic and oncotic aneurysms. Interv. Neuroradiol. 22, 524-528. doi: 10.1177/1591019916653247

Winquist, R. J., and Bohr, D. F. (1983). Structural and functional changes in cerebral arteries from spontaneously hypertensive rats. Hypertension 5, 292-297. doi: 10.1161/01.HYP.5.3.292

Wu, X. P., Ni, J. M., Zhang, Z. Y., Lu, F. Q., Li, B., Jin, H. H., et al. (2015). Preoperative evaluation of malignant perihilar biliary obstruction: negative-contrast CT cholangiopancreatography and CT angiography versus MRCP and MR angiography. AJR Am. J. Roentgenol. 205, 780-788. doi: 10.2214/AJR.14.13983

Xi, Y., Lin, X., Yuan, F., Yang, G. Y., and Zhao, J. (2015). High-resolution and quantitative X-Ray Phase-contrast tomography for mouse brain research. Comput. Math. Methods Med. 2015:530580. doi: 10.1155/2015/530580

Xu, J., Chen, A., Xiao, J., Jiang, Z., Tian, Y., Tang, Q., et al. (2016). Evaluation of tumour vascular distribution and function using immunohistochemistry and BOLD fMRI with carbogen inhalation. Clin. Radiol. 71, 1255-1262. doi: 10.1016/j.crad.2016.04.012

Yang, Y. M., Feng, X., Yao, Z. W., Tang, W. J., Liu, H. Q., and Zhang, L. (2008). Magnetic resonance angiography of carotid and cerebral arterial occlusion in rats using a clinical scanner. J. Neurosci. Methods 167, 176-183. doi: 10.1016/j.jneumeth.2007.08.005
Yuan, F., Lin, X., Guan, Y., Mu, Z., Chen, K., Wang, Y., et al. (2014). Collateral circulation prevents masticatory muscle impairment in rat middle cerebral artery occlusion model. J. Synchrotron Radiat. 21, 1314-1318. doi: 10.1107/S1600577514016130

Yuan, F., Wang, Y., Guan, Y., Ren, Y., Lu, H., Xiao, T., et al. (2013). Real-time imaging of mouse lenticulostriate artery following brain ischemia. Front. Biosci. (Elite. Ed). 5, 517-524. doi: 10.2741/E633

Zhang, M., Peng, G., Sun, D., Xie, Y., Xia, J., Long, H., et al. (2014). Synchrotron radiation imaging is a powerful tool to image brain microvasculature. Med. Phys. 41:031907. doi: 10.1118/1.4865784

Conflict of Interest Statement: The authors declare that the research was conducted in the absence of any commercial or financial relationships that could be construed as a potential conflict of interest.

Copyright (c) 2017 Wang, Mu, Lin, Geng, Xiao, Zhang, Wang, Guan and Yang. This is an open-access article distributed under the terms of the Creative Commons Attribution License (CC BY). The use, distribution or reproduction in other forums is permitted, provided the original author(s) or licensor are credited and that the original publication in this journal is cited, in accordance with accepted academic practice. No use, distribution or reproduction is permitted which does not comply with these terms. 